\title{
ESTUDOS CTS NA EDUCAÇÃO CIENTÍFICA: tendências e perspectivas da produção stricto sensu no Nordeste brasileiro
}

\author{
Marcelo Bruno Araújo-Queiroz ${ }^{1}$ \\ Rodrigo da Luz Silva² \\ Christiana Andréa Vianna Prudêncio 3
}

\section{RESUMO}

Apresentamos dados teóricos sobre a produção de dissertações e teses que incorporaram a Educação CTS como temática de pesquisa no Ensino de Ciências no Nordeste brasileiro. Esse recorte, composto por 35 pesquisas defendidas entre 2007 e 2017, considera cinco categorias: produção por estado, produção por programa de pós-graduação, perspectiva curricular abordada, contexto da pesquisa e área de conhecimento. A partir da análise dos resumos dos trabalhos discutimos as perspectivas e tendências dessa produção que se mostra em processo de expansão e possivelmente poderá se consolidar na região. Além disso, trazemos informações que podem contribuir para um processo de implementação dessa perspectiva de educação no ensino, no currículo e na formação de professores de Ciências.

Palavras-chave: Ciência-Tecnologia-Sociedade. Educação em Ciências. Mestrado/Doutorado.

\section{STS STUDIES IN SCIENTIFIC EDUCATION: tendencies and perspectives of stricto sensu production in Northeast Brazil}

\begin{abstract}
We present theoretical data on the production of dissertations and theses that incorporated the STS Education as a research theme in Science Education in Northeast Brazil. This sample, composed of 35 studies defended between 2007 and 2017, considers five categories: production by state, production by postgraduate program,

\footnotetext{
1 Licenciado em Ciências Biológicas (UFPI) e mestrando em Educação em Ciências (UESC). Integrante do grupo de estudo/pesquisa em "Temas atuais para o ensino em Ciências". E-mail: marcelobrunoqueiroz@gmail.com

2 Licenciado em Biologia (UFRB) e mestrando em Educação em Ciências (UESC). Integrante do grupo de estudo/pesquisa em "Temas atuais para o ensino em Ciências". E-mail: rodrigoluz_saj@live.com

3 Mestre e doutora em Educação pela Universidade Federal de São Carlos/SP - UFSCar. Professora adjunta da Área de Ensino de Biologia da Universidade Estadual de Santa Cruz (UESC) e membro permanente do Programa de Pós-Graduação em Educação em Ciências dessa Universidade. Líder do grupo de estudo/pesquisa em "Temas atuais para o ensino em Ciências". E-mail: cavprudencio@vesc.br
} 
curricular perspective, research context and area of knowledge. From the analysis of the summaries of the works, we discuss the perspectives and tendencies of this production that shows itself in a process of expansion that possibly will be consolidated in the region. In addition, we bring information that may contribute to a process of implementation of this educational perspective in teaching, curriculum and in the training of science teachers.

Keywords: Science-Technology-Society. Education in Sciences. Masters/Doctorate degree.

\section{ESTUDIOS CTS EN LA EDUCACIÓN CIENTÍFICA: tendencias y perspectivas de la producción stricto sensu en el Nordeste brasileño}

\section{RESUMEN}

Presentamos datos teóricos sobre la producción de disertaciones y tesis que incorporaron la Educación CTS como temática de investigación en la Enseñanza de Ciencias en el Nordeste brasileño. Este recorte, compuesto por 35 investigaciones defendidas entre 2007 y 2017, considera cinco categorías: producción por Estado, producción por programa de postgrado, perspectiva curricular abordada, contexto de la investigación y área de conocimiento. A partir del análisis de los resúmenes de los trabajos discutimos las perspectivas y tendencias de esa producción que se muestra en proceso de expansión y posiblemente podrá consolidarse en la región. Además, presentamos información que puede contribuir a un proceso de implementación de esa perspectiva de educación en la enseñanza, en el currículo y en la formación de profesores de Ciencias.

Palabras clave: Ciencia-Tecnología-Sociedad. Educación en Ciencias. Maestría/Doctorado.

\section{CONTEXTUALIZANDO}

A Educação CTS (Ciência-Tecnologia-Sociedade) é uma perspectiva curricular que vem se consolidando nos últimos 20 anos no campo da Educação Científica ${ }^{4}$ no Brasil (FREITAS; GHEDIN, 2015). Desde a década de 1990 essa proposta tem contribuído para o rompimento dos métodos e técnicas de ensino tradicionais e propedêuticos, buscando (re)orientar o currículo de Ciências a partir da abordagem de temas ou situações reais socialmente relevantes.

Deste modo, tem direcionado alunos e professores - que chamaremos aqui de cidadãos - a terem uma visão crítica sobre o ensino de Ciências e sobre o papel deste na sociedade. Nesse sentido, a Educação CTS almeja a

${ }^{4}$ O termo Educação Científica será utilizado como sinônimo do termo Educação em Ciências. 
formação crítica do sujeito para o exercício da cidadania, buscando promover o desenvolvimento de valores e atitudes (SANTOS; MORTIMER, 2000) para resolver e discutir problemas sociais oriundos do desenvolvimento científicotecnológico, como também ampliar sua participação social (AULER; 2002) para a tomada de decisões sobre assuntos relacionados à ciência e à tecnologia (SANTOS; MORTIMER, 2000, 2001).

As discussões iniciais sobre as inter-relações entre Ciência, Tecnologia e Sociedade surgiram em meados das décadas de 1960 e 1970 em países da Europa e da América do Norte, sendo intitulado inicialmente como um Movimento de participação pública (AULER, 2002), externo ao contexto educacional. As inquietações começaram a emergir após a Segunda Guerra Mundial (1939-1945), quando os países envolvidos preconizavam a formação de cientistas a todo custo para o desenvolvimento e produção de novas armas ou aparelhos tecnologicamente mais potentes (SANTOS; MORTIMER, 2001; AIKENHEAD, 2003; SANTOS, 2011), não se preocupando com as consequências do desenvolvimento da Ciência e da Tecnologia para a sociedade. Esse movimento repercutiu também devido ao agravamento de sérios problemas ambientais e pela necessidade da participação popular nas decisões públicas (SANTOS; MORTIMER, 2000).

No contexto brasileiro, e especialmente no ensino de Ciências, essas discussões começaram somente na década de 1990. Em 1992 a primeira pesquisa acadêmica sobre CTS ocorreu no âmbito do ensino de Química e foi desenvolvida por Wildson Santos que inicia os estudos no campo com sua dissertação de mestrado, seguido pela tese de doutorado de Silvia Trivelato (1993) e a dissertação de Antônio Carlos Amorim (1995). Já na década de 2000, duas importantes e referenciadas pesquisas nos primeiros anos são publicadas: o artigo de Santos e Mortimer (2000) sobre os pressupostos teóricos da abordagem CTS no contexto da educação brasileira e a tese de doutorado de Auler (2002) sobre interações CTS na formação de professores.

A partir desse período, diversos pesquisadores passaram a compreender os pressupostos da Educação CTS como norteadores de uma educação crítica e autônoma, capaz de contribuir para o processo de reflexão e 
desenvolvimento de uma consciência integradora que possibilitasse 0 desvelamento do mundo. A ascensão desse novo campo de conhecimento no Brasil gerou um crescimento expressivo de pesquisas sobre a temática nos últimos 15 anos. Segundo Strieder (2012), esse aumento significativo tornou CTS um campo polissêmico no contexto brasileiro.

Muitas pesquisas adotam práticas que divergem dos objetivos originais inerentes ao movimento de Educação CTS e defendem novas possibilidades de implementações e/ou discussões teóricas sobre essa perspectiva. Não é nosso objetivo aqui nos atermos a essas situações, mas corroborar com Santos (2011) quando afirma que a Educação CTS constitui-se como um campo novo e emergente, longe de ser ultrapassado, e que, devido a diversos direcionamentos dados pelos pesquisadores, necessita ser ressignificado.

Os pesquisadores dos países da América Latina preocupados com a realidade vigente desses países articularam uma visão diferenciada e a denominaram Pensamento Latino Americano em Ciência-TecnologiaSociedade (PLACTS), que diverge em alguns aspectos das visões europeia e norte-americana, origens desse movimento. Uma característica marcante do PLACTS é a preocupação em atender, discutir e problematizar aspectos relacionados às necessidades regionais onde estão inseridos os indivíduos. Segundo Dagnino, Thomas e Davył (2003) a estratégia implementada responde de forma contrária ao modelo linear de desenvolvimento científico e tecnológico e refuta seus fundamentos e pressupostos. Para Strieder (2012), o PLACTS também se caracteriza como uma crítica ao modelo de política científico-tecnológica adotado nos países latino-americanos que se baseia em países de "Primeiro Mundo", negligenciando as necessidades regionais.

No Brasil esse pensamento ganha repercussão no ensino de Ciências ancorado a uma visão crítica de Educação CTS que tem como base os ideais de Paulo Freire. O PLACTS tem incorporado os pressupostos educacionais freireanos na medida em que visa superar os mitos ligados ao desenvolvimento científico tecnológico (AULER; 2007), além de contribuir para mudanças significativas nas agendas de pesquisa sobre Educação CTS no cenário latinoamericano. É dessa maneira que a concepção de Santos (2011), ao propor 
uma ressignificação para a Educação CTS, pode ser estabelecida no que diz respeito a problematizar as relações CTS com aportes freireanos no intuito de estabelecer-se uma educação científica humanística (SANTOS, 2008, 2009, 2012).

Desta maneira, diversos programas de pós-graduação (PPG) stricto sensu no Brasil começaram a desenvolver estudos e pesquisas sobre CTS/CTSA 5 e suas contribuições para a educação, em linhas de pesquisa sobre formação de professores, currículo e processos de ensino e aprendizagem. Com destaque nesses estudos, pode-se citar o Programa de Pós-Graduação em Educação Científica e Tecnológica da Universidade Federal de Santa Catarina - UFSC (região Sul) com uma linha de pesquisa específica para estudos CTS, além de significativa produção de dissertações e teses na área. Ainda não é comum um PPG em Ensino de Ciências ter a Educação CTS como uma linha de pesquisa específica, em contrapartida, essa temática tem sido discutida para além das pesquisas, sendo proposta como disciplina em cursos de graduação e pós-graduação onde diversos grupos de estudos/pesquisas consolidados estão ativos e certificados.

Já estão publicados alguns levantamentos sobre a produção CTS no Brasil (ABREU; FERNANDES; MARTINS, 2013; FREITAS; GHEDIN, 2015; ARAUJOQUEIROZ; MENDES; ARAÚJO, 2016; PINTO; GOULART; VERMELHO, 2016; SILVA et al, 2016). Outros levantamentos ganham destaque em nosso estudo por serem realizados com informações da região Nordeste. Gonçalves e Silva (2016), por exemplo, fizeram um mapeamento das pesquisas que abordam CTS no âmbito de PPG stricto sensu do Nordeste com nota igual ou superior a quatro na avaliação da Coordenação de Aperfeiçoamento de Pessoal de Nível Superior (CAPES). Já Failache et al, (2017), articularam uma investigação sobre a presença e o desenvolvimento de programas CTS ou de programas que continham algum viés crítico e reflexivo que se aproximava de CTS na região Nordeste.

\footnotetext{
5 Ciência-Tecnologia-Sociedade-Ambiente. Alguns pesquisadores utilizam CTSA com o intuito de explicitar e "valorizar" as questões ambientais. Esses mesmos autores defendem o uso da letra "A" ao final da tríade CTS, por entenderem que essas questões ficam excludentes quando o processo intitula-se por CTS (ver, por exemplo, VILCHES; PÉREZ; PRAIA, 2011; SANTOS, 2011).
} 


\section{ISSN 2237-9460}

Entretanto, tais pesquisas foram desenvolvidas com discussões ainda incipientes. O primeiro levantamento traz apenas 10 trabalhos para análise, sendo que em nossa investigação encontramos $35 \mathrm{em}$ um período de dez anos. O que se pode notar é que os autores utilizaram descritores que limitaram o real "estado da arte" das pesquisas. Além disso, não contabilizaram alguns trabalhos e utilizaram como critérios de análise apenas a área de conhecimento e o enfoque da pesquisa.

A segunda pesquisa que destacamos traz poucas contribuições especificamente para a Educação Científica, isso porque os pesquisadores investigam CTS no campo da Tecnologia e da Comunicação, em uma linha de trabalho divergente da proposta na área. Além disso, obtêm as informações da pesquisa em PPG que não só trabalham com Educação e Educação em Ciências, mas Cultura e Sociedade, Comunicação e Sociedade etc.

No Nordeste, em especial na pós-graduação stricto sensu, pesquisas sobre CTS começaram a ser desenvolvidas aproximadamente nos anos de 2005 e 2006, sendo que a primeira dissertação foi defendida em 2007 por Ruth Firme. Após esse período, somente em 2010 é que se iniciam de fato as discussões sobre essa perspectiva de modo mais amplo e em 2012 ocorre a defesa da primeira tese sobre a temática, desenvolvida também pela pesquisadora supracitada.

Afinal, por que analisar a produção CTS no Nordeste brasileiro? É muito comum encontrar trabalhos sobre essa temática em periódicos nacionais veiculados por pesquisadores das regiões Sul, Sudeste e Centro-Oeste, enquanto na região Nordeste essa produção não é tão percebida. Partindo da ideia de que grande parte dessas publicações se origina de resultados de dissertações e teses, decidimos fazer um recorte considerando a realidade nordestina brasileira para saber como vem se desenvolvendo o campo de estudos CTS em seus PPG, levando em consideração o fato de que os programas em Ensino de Ciências ainda estão se consolidando.

É importante ressaltar que os dois primeiros autores da presente pesquisa possuem raízes formativas na região Nordeste e percebem que existem poucas pesquisas sobre CTS em nível de pós-graduação que considerem essa região e 
suas especificidades acadêmicas, como mostra esse levantamento. Dessa forma, decidimos não restringir essa pesquisa somente à área de Ensino, mas considerar também a Educação, justamente para mapearmos todos os trabalhos voltados a processos educativos. Esses dados ajudam-nos a apresentar um panorama do campo de discussões da Educação CTS no Nordeste.

Pensando no cenário nordestino, decidimos investigar como vem sendo desenvolvidas as pesquisas sobre essa temática nos PPG em Educação e Ensino de Ciências presentes nessa região do Brasil. Buscamos trazer respostas para os seguintes questionamentos: 1) quais PPG stricto sensu de Educação e Ensino de Ciências no Nordeste discutem CTS?; 2) qual o quantitativo de pesquisas CTS por estado e por PPG?; 3) como se caracterizam essas produções em relação à perspectiva adotada e qual seu contexto de pesquisa?; e 4) em quais áreas do conhecimento estão sendo desenvolvidas essas pesquisas?

\section{TRAÇANDO CAMINHOS: onde está a Educação Cts do Nordeste?}

Realizamos um levantamento das dissertações e teses em 28 PPG stricto sensu em Educação e Ensino de Ciências das universidades do Nordeste. Existem ao todo $32^{6}$ PPG com essa filiação em funcionamento na região, no entanto, quatro deles não participaram de nossa investigação, porque iniciaram suas atividades em 2015 e até o momento do levantamento desses dados, não possuíam nenhuma dissertação defendida, como o PPG em Ensino de Ciências e Matemática da Universidade Federal do Maranhão (UFMA) e o de Educação da Universidade Federal de Campina Grande (UFCG) na Paraíba.

Consideramos também os PPG em Educação por entendermos que representam espaços em que se discute sobre Educação Científica independentemente de possuírem uma linha específica para isso. Um exemplo é o PPG em Educação da Universidade Federal do Piauí (UFPI), que não possui

\footnotetext{
${ }^{6}$ Ressaltamos que esse número corresponde somente aos programas que se filiam a Educação ou Ensino de Ciências. Alguns programas em Educação que possuem área de concentração ou filiam-se a outras áreas, como, por exemplo, Educação e Cultura, Educação e Diversidade, Educação e Contemporaneidade, não participaram do levantamento.
} 
uma linha de pesquisa específica sobre Educação CTS, mas possui professores credenciados que pesquisam no campo da Educação em Ciências na linha de pesquisa "Ensino, Práticas Pedagógicas e Formação de Professores", contribuindo com diversos trabalhos para o ensino e a formação de professores de Ciências.

Como dito anteriormente, alguns PPG foram excluídos da análise, mas, ressaltamos que há pesquisas em pleno desenvolvimento sobre Educação CTS no Nordeste nesses programas novos. O PPG em Ensino da Universidade Estadual do Rio Grande do Norte (UERN), por exemplo, não contabilizou na presente investigação, porque iniciou suas atividades no segundo semestre de 2015. No entanto, no calendário de qualificações da primeira turma já identificamos três pesquisas referentes à Educação CTS, com diferentes professores orientadores. O mesmo acontece com o PPG em Educação em Ciências da Universidade Estadual de Santa Cruz (UESC) que não entrou para análise por não possuir nenhuma pesquisa concluída sobre CTS, ainda que quatro dissertações sobre o tema estejam em pleno desenvolvimento no momento da obtenção dessas informações. De certa forma, os programas específicos em Ensino de Ciências ainda são novos no Nordeste e as pesquisas sobre CTS ainda estão se consolidando nesta região. No entanto, a variedade de professores orientadores nesta linha de pesquisa mostra o engajamento e o interesse dos mesmos em investigações que envolvam a Educação CTS.

Encontramos os trabalhos disponíveis nos sites dos programas, na plataforma Sucupira e/ou no repositório de dissertações e teses das instituições formadoras. Para a busca destes consideramos os termos "CTS, C.T.S, C/T/S e CT-S" abreviado ou por extenso nos títulos, resumos e palavras-chave dos trabalhos. Consideramos o período de 10 anos (2007-2017), porque a primeira pesquisa finalizada no nordeste ocorreu em 2007, nesse sentido, conseguimos analisar todo o panorama regional da área.

Selecionamos 35 pesquisas defendidas, sendo 33 oriundas de cursos de mestrado e duas de doutorado. Os trabalhos de mestrado foram de autoria de Firme (2007), Nunes (2010), Oliveira (2010), Souza (2010), Dantas (2011), Lima Neto (2012), Oliveira (2012), Silva (2012), Bitencourt (2013), Oliveira (2013), Sousa 
(2013), Andrade (2016), Bezerra (2014), Macedo (2014), Marques (2014), Porto (2014), Rosa (2014), Santana (2014), Silva (2014), Binatto (2015), Costa (2015), Andrade Junior (2015), Santos, A. (2015), Santos, E. (2015), Almeida (2016), Gomes (2016), Santana (2016), Santiago (2016), Silva, D. (2016), Silva, M. (2016), Jesus (2017), Santos, M. (2017) e Santos, R. (2017).

Já as investigações em nível de doutorado tiveram autoria de Ruth Firme (2012) e Albino Nunes (2014). Apesar desta segunda tese ter sido desenvolvida em um PPG que não pertence ao eixo da Educação e do Ensino (o PPG em Química da Universidade Federal do Rio Grande do Norte), a contabilizamos para a pesquisa por ser voltada para a temática de Ensino de Ciências e tratar da Educação CTS, constituindo a segunda tese do Nordeste.

Nessa pesquisa delimitamos a análise aos PPG em Educação e Ensino de Ciências ${ }^{7}$. No entanto, no momento do levantamento dos trabalhos, pesquisamos nas bases de dados de forma ampla, sem especificar tais programas, o que nos permite afirmar que filtramos todos os PGG do Nordeste. Elencamos cinco categorias de análise que emergiram a partir de nossas questões de pesquisa e utilizamos a leitura dos resumos para categorizar cada trabalho.

\section{CARACTERIZANDO TENDÊNCIAS E PERSPECTIVAS NAS DISSERTAÇÕES E TESES: a} Educação CTS em foco

A região Nordeste possui 17 PPG em Educação (15 intitulados por Educação, um intitulado por Educação Profissional e outro por Educação e Ensino) e 13 programas de Ensino de Ciências e Matemática (sendo alguns somente de Ciências, mas com área de avaliação em Ensino de Ciências e Matemática na CAPES).

Elencamos cinco categorias de análise para descrever as tendências e perspectivas da produção CTS nessa região. As categorias compreendem: a) produção CTS por estado; b) produção por programa de pós-graduação; c) perspectiva curricular abordada; d) contexto que se inserem as pesquisas; e e) área do conhecimento da pesquisa na Educação Científica.

\footnotetext{
7 Utilizamos o termo Ensino de Ciências para caracterizar todos os PPG que estão na área de avaliação em Ensino de Ciências e Matemática da CAPES.
} 


\section{Produção por estado}

Localizamos 35 pesquisas sobre a temática CTS nos PPG do Nordeste. Essa distribuição por estado quantifica oito produções na Bahia, cinco na Paraíba, três em Pernambuco, doze no Rio Grande do Norte e sete em Sergipe. A figura 1 mostra a distribuição em porcentagem (\%).

Figura 01. Distribuição quantitativa por estado da produção de pesquisas sobre Educação CTS no período de 2007 a 2017

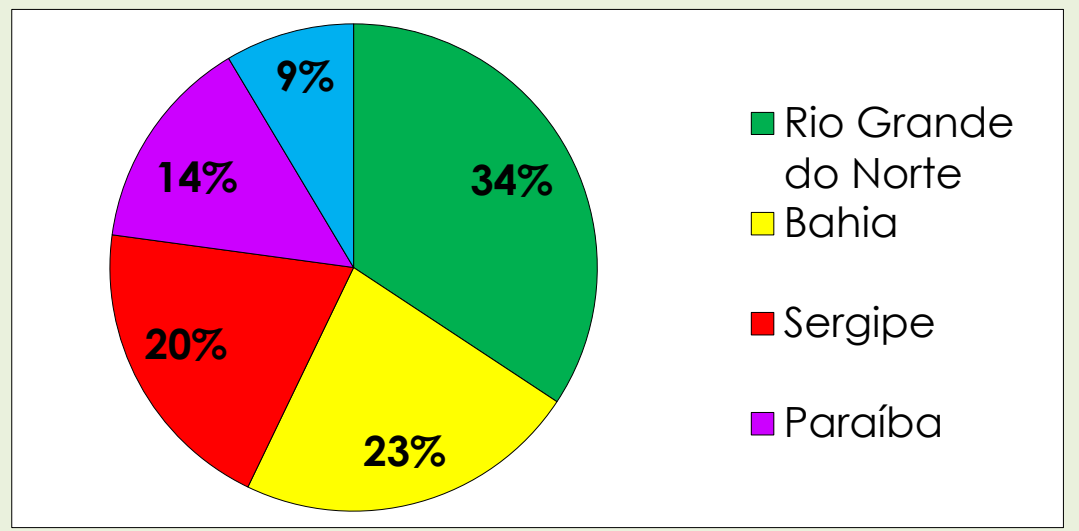

Fonte: dados da pesquisa, 2017.

Pela figura 01 podemos perceber o quanto essa perspectiva ainda é carente de investigações nesta região do país, revelando que os estados do Maranhão, Piauí, Ceará e Alagoas ainda não possuem produção nessa temática. Os possíveis motivos para isso encontram-se nos próprios PPG das universidades presentes nesses estados, que não possuem professores que trabalhem com essa proposta, como mostra o quadro de docentes nos sites destes. Dentre esses quatro estados, destaca-se o Maranhão com um PPG em Ensino de Ciências e Matemática, onde existe uma linha de pesquisa específica denominada "Ciência, Tecnologia e Sociedade", entretanto, sem nenhuma pesquisa concluída até o momento desse levantamento.

Freitas e Ghedin (2015) consideram que o campo CTS vem se consolidando no Brasil devido à quantidade expressiva de artigos publicados em periódicos da área de Educação em Ciências. Apesar de sabermos que a produção sobre determinado campo de conhecimento não se restringe a dissertações e teses desenvolvidas em PPG, a pouca abrangência da 
Educação CTS nos estados do Nordeste revela que as investigações nessa área podem estar ainda muito concentradas na região Sul, Sudeste e Centro-Oeste, havendo a necessidade de ampliar as pesquisas para outras regiões do país de modo a se obter uma consolidação mais expressiva.

Na Figura 01 é possível verificar ainda que o estado do Rio Grande do Norte lidera em quantidade de pesquisas sobre Educação CTS, sendo que durante esse período de dez anos, doze investigações foram desenvolvidas, sendo uma delas de doutorado. De certo modo não podemos afirmar com precisão o porquê dessa quantidade de pesquisas considerada expressiva nesse estado, mas é importante ressaltar que o PPG em Ensino de Ciências Naturais e Matemática (PPGECNM) da Universidade Federal do Rio Grande do Norte (UFRN) iniciou suas atividades em 2002, período em que a perspectiva curricular CTS estava se estruturando como campo de pesquisa no Brasil.

Porém, ressaltamos que esse número significativo está relacionado às afinidades de pesquisas dos professores credenciados no programa. Realizamos um mapeamento dos professores orientadores no PPGECNM/UFRN e detectamos que os docentes que trabalham com CTS orientaram, cada um, mais de dois trabalhos e continuam realizando orientações, como é possível verificar em seus currículos na plataforma lattes. É necessário evidenciar também que estes pesquisadores não possuem raízes formativas na Educação em Ciências, como revela essa verificação em seus currículos. Isso ainda é muito comum entre os professores que orientam pesquisas sobre CTS no Nordeste e, principalmente, em PPG profissionais, o que pode contribuir de forma significativa para que o campo da Educação CTS se torne ainda mais polissêmico.

Strieder e Kawamura (2017) destacam que os espaços pedagógicos de desenvolvimento de propostas CTS e a complexidade das próprias questões CTS ao serem implementadas no contexto educacional podem ser fatores que reforçam essa polissemia, porque envolvem uma série de variáveis que perpassam diferentes campos do conhecimento, dando margem a recortes variados. Esses fatores podem ser levados em consideração pelo fato dos trabalhos analisados apresentarem caráter interventivo. 
O PPG pioneiro na produção sobre CTS foi o de Educação nas Ciências da Universidade Federal Rural do Pernambuco (UFRPE), que teve a primeira dissertação defendida em 2007. O programa iniciou suas atividades em 1995, porém, possui ainda hoje uma baixa produção de pesquisa com essa temática, pelo fato dos professores credenciados terem como interesse outras linhas de investigação.

$\mathrm{Na}$ Bahia o maior número de pesquisas sobre CTS concentra-se na Universidade Estadual do Sudoeste da Bahia (UESB), campus de Jequié. O PPG em Educação Científica e Formação de Professores - PPGECFP possui um grupo de pesquisadores que investiga a Educação CTS no ensino e na formação de professores. Apesar de o programa ser relativamente recente, tendo iniciado suas atividades em 2011, já possui um grupo de pesquisa consolidado sobre essas discussões, liderado pelo professor Paulo Marcelo Marini Teixeira, considerado um dos referenciais de CTS, sendo citado em pesquisas e realizando palestras que tratam dessa perspectiva. Além disso, essas pesquisas concentram-se no ensino de Biologia, característica pouco visualizada em outros PPG no Brasil. Foi no PPGECFP que foram divulgadas as duas primeiras pesquisas sobre CTS na Bahia, no ano de 2013. Até então, o programa tem contribuído com discussões sobre a temática e divulgado seus resultados em periódicos nacionais e eventos da área.

No estado de Sergipe também vêm sendo desenvolvidas pesquisas sobre CTS e até o momento da presente pesquisa foram encontrados sete trabalhos, sendo três defendidos em 2017. As produções são oriundas do PPG na área de Ensino de Ciências. Já o PPG da Paraíba apresenta cinco pesquisas sobre a temática, sendo as duas mais recentes defendidas em 2015. Os professores que orientam sobre CTS no programa também não concentram seus interesses de pesquisa nessa temática. Contudo, de modo geral, podemos considerar que as pesquisas na região Nordeste sobre CTS vêm avançando, mesmo que em passos lentos.

\section{Produção por programa de pós-graduação}

O quadro 1 apresenta o quantitativo da produção acadêmica sobre Educação CTS encontrada nos PPG presentes na região Nordeste. Ressalta-se 
que na coluna sobre produção CTS as células em destaque contabilizam as duas teses de doutorado defendidas, sendo os demais referentes a trabalhos de dissertação.

QUADRO 01. Pesquisas sobre CTS desenvolvidas por programa de pósgraduação entre 2007 e 2017

\begin{tabular}{|l|c|c|c|}
\hline \multicolumn{1}{|c|}{ Estado } & IES & Programa(s) & Produção CTS \\
\hline \multirow{2}{*}{ Bahia } & UESB & PPGECFP & 6 \\
\cline { 2 - 4 } & UFBA & PPGEHFC & 2 \\
\hline Paraíba & UEPB & PPGECM & 5 \\
\hline \multirow{2}{*}{ Pernambuco } & UFPE & PPGE & 1 \\
\cline { 2 - 4 } & UFRPE & PPGEC & 2 \\
\hline Rio Grande do Norte & \multirow{2}{*}{ UFRN } & PPGECNM & 11 \\
\cline { 2 - 4 } & & PPGQ & 1 \\
\hline Sergipe & UFS & PPGECIMA & 7 \\
\hline TOTAL & \multicolumn{2}{|c|}{} \\
\hline
\end{tabular}

Fonte: dados da pesquisa, 2017.

Podemos perceber que a produção por PPG no Nordeste ainda é pequena principalmente se considerarmos a produção dos PPG das regiões Sul, Sudeste e Centro-Oeste. As pesquisas com essa temática em PPG de Educação geralmente só acontecem de forma contínua em um programa que possui o Ensino de Ciências como linha de pesquisa. Isso não é comum no Nordeste, uma vez que apenas o PPG em Educação da Universidade Federal do Ceará (UFC) possui uma linha de pesquisa específica em Ensino de Ciências. No entanto, há diversos professores credenciados em programas de Educação que orientam pesquisas da Educação Científica em linhas de investigação mais abrangentes, como formação docente e práticas pedagógicas, por exemplo.

Das 35 produções encontradas no Nordeste, uma encontra-se em um PPG de Educação que é a tese de doutorado de Ruth Firme (2012), desenvolvida no âmbito do PPG em Educação (PPGE) da Universidade Federal do Pernambuco - UFPE. Um dos motivos que deve ter levado a autora a fazer o doutorado no PPG supracitado pode ter sido à inexistência de um PPG em nível de doutorado na área de Ensino de Ciências no estado do Pernambuco,

\footnotetext{
8 Ressaltamos que essas informações foram minuciosamente analisadas e filtradas. A obtenção desses dados nos locais de pesquisa foi realizada até junho de 2017. Trabalhos apresentados em período anterior a essa data e publicados nos locais de busca estão contabilizados. Aqueles que não disponibilizaram a versão final para inclusão e publicação nas plataformas de arquivamento de dissertações e teses não foram incluídos.
}

Revista Exitus, Santarém/PA, Vol. 8, №3, p. 310 - 339, SET/DEZ 2018. 
quando esta finalizou o mestrado em Ensino das Ciências na UFRPE. O programa só foi criado dois anos após seu ingresso no doutorado. Essa foi a mesma razão de Nunes9 (2014) que realizou seu doutorado em um PPG específico da Química, mas, com co-orientação de duas pesquisadoras que atuam na Educação em Ciências/Química, desenvolvendo, por sua vez, a segunda pesquisa de Doutorado sobre CTS no Nordeste. Isso nos remete dizer que especificamente nos PPG em Ensino de Ciências na região ainda não foi desenvolvida nenhuma pesquisa de doutorado sobre Educação CTS, uma vez que as duas existentes se originaram em um contexto de "ausência" de um PPG específico.

Essa tendência de migrar para outro PPG sem uma linha específica na área de Ensino de Ciências está diminuindo devido à quantidade de PPG específicos que vem se expandindo e se consolidando na área. Nesse âmbito, ainda é muito comum professores com pós-graduação em outra área (geralmente áreas específicas das Ciências) atuarem em PPG em Ensino de Ciências. Ao tempo que defendemos que os pesquisadores para atuarem na Educação em Ciências devam ter formação na área, consideramos que presença de doutores nesse campo ainda é inexpressiva, contudo, isso vem diminuindo com o estabelecimento de novos programas e com a formação de novos profissionais com essa titulação.

Nesse sentido, Barolli e Villani (2015) apresentam críticas aos mestrados profissionais ao discutirem a formação de professores como um campo de disputa entre as instituições governamentais; a instituição científica (composta pelos pesquisadores de áreas específicas das ciências naturais e por pesquisadores da área de ensino de ciências naturais) e a instituição escolar. Os pesquisadores argumentam que:

O documento da CAPES que regulamenta esses mestrados, ao caracterizar a competência dos docentes que neles atuam, não fazem nenhuma consideração a respeito da necessidade desse profissional possuir uma vivência e uma prática originais e efetivas na área educacional. [...] Esse lapso, digamos assim, abre a possibilidade para que profissionais das áreas específicas das ciências possam implícita e explicitamente orientar o desenvolvimento profissional dos professores. [...] Como pode-se observar nos sites correspondentes desses mestrados,

\footnotetext{
9 Em contato com o pesquisador/professor essas informações foram validadas.
} 
observa-se que nos dois casos a ênfase do desenvolvimento profissional está voltada prioritariamente para o domínio aprofundado dos conteúdos das respectivas áreas (BAROLLI; VILLANI, 2015, p. 87).

Ressalta-se também, que muitas vagas da área de ensino nas universidades ainda estão efetivamente preenchidas por pesquisadores da área específica em Ciências, justamente pelo fato da formação de mestres e doutores em Ensino de Ciências ser recente, principalmente no Nordeste, acarretando inclusive a migração de pesquisadores de outras regiões do país geralmente Centro-Oeste, Sul e Sudeste - para ocuparem essas vagas de ensino via concurso público. Todos esses fatores podem refletir na produção de pesquisas sobre Educação CTS nos PPG analisados.

O PPG pioneiro na área de Ensino de Ciências no Nordeste é o da Universidade Federal da Bahia - UFBA. Esse programa iniciou suas atividades de mestrado em 1972 e de doutorado em 1992. É o programa com maior conceito no Nordeste, sendo avaliado com nota 5 pela CAPES10. Contudo, só localizamos duas pesquisas na temática CTS/CTSA nesse programa, ambas defendidas em 2016. No entanto, os pesquisadores após terem concluído o mestrado ingressaram no doutorado no mesmo programa e com a mesma temática de pesquisa, ou seja, brevemente novas pesquisas de doutorado serão veiculadas. O nome "Programa de Pós-Graduação em Ensino, História e Filosofia das Ciências - PPGEHFC" acaba, por sua vez, designando outras possibilidades e vieses de pesquisa que podem não contemplar, neste caso, propostas para discussões sobre o campo de conhecimento CTS.

Não é nossa intenção caracterizar quais PPG desenvolveram mais ou menos pesquisas, mas expor as contribuições de cada programa para a difusão da pesquisa com a temática CTS nos últimos dez anos na região Nordeste. Entendemos que a tendência de pesquisa nessa temática está em crescimento considerável, tendo em vista as especificidades dos programas de pós-graduação discutidas até aqui. Nesse sentido, e de acordo com a produção da região e dos programas, é viável afirmar que os números desse levantamento poderão crescer significativamente a partir de 2020, momento

10 Avaliação Quadrienal de 2017.

Revista Exitus, Santarém/PA, Vol. 8, №3, p. 310 - 339, SET/DEZ 2018. 


\section{ISSN 2237-9460}

em que novas pesquisas estarão sendo defendidas e, possivelmente, publicadas.

\section{Perspectiva curricular abordada}

Comumente temos encontrado dois slogans para caracterizar as perspectivas curriculares sobre a temática científico-tecnológica no ensino de Ciências: CTS e CTSA. A Educação CTS é usual para pesquisadores que entendem que as questões ambientais já estão implícitas na tríade CTS (SANTOS, 2011, 2012; STRIEDER, 2012). Outros grupos de pesquisadores utilizam a sigla CTSA para dar maior ênfase à perspectiva ambiental, acrescentando a letra "A" para caracterizar o Ambiente (VILCHES; GIL PÉREZ; PRAIA, 2011), buscando resgatar o papel da educação ambiental (SANTOS, 2007) que muitos cursos CTS não contemplaram (SANTOS, 2011).

Antes da perspectiva curricular CTS passar por um processo de inserção no contexto educacional, ela já estava consolidada como um movimento de participação pública no contexto norte-americano e europeu. O surgimento desse movimento decorreu com uma forte crítica ao modelo econômico desenvolvimentista com elevado impacto ambiental e sobre o papel da ciência na sociedade (SANTOS; MORTIMER, 2000; SANTOS, 2011). Auler (2002) descreve que duas grandes obras publicadas em 1962 marcaram o surgimento do movimento CTS: o livro A Estrutura das Revoluções Científicas, do físico Thomas Kuhn e Silent Spring (Primavera Silenciosa) da bióloga naturalista Rachel Carson.

Esse segundo livro constituiu-se como um marco de fundamental importância para a origem do movimento CTS, pois mostra que desde o início o movimento tem se preocupado com as questões ambientais. É partindo desse argumento que sustentamos o pressuposto de que a Educação CTS já incorpora aspectos ambientais na discussão das inter-relações Ciência, Tecnologia e Sociedade e, desse modo, não necessita evidenciar essa preocupação ambiental agregando a letra "A" à tríade. No entanto, é importante ressaltar que entendemos que utilizar uma temática ambiental como "pano de fundo" ou somente para iniciar uma discussão CTS, sem 
problematizá-la em seus aspectos multidimensionais, pode remeter a uma concepção ingênua e a um discurso vazio sobre meio ambiente.

Vilches, Gil Pérez e Praia (2011) argumentam que a incorporação da letra "A" na tríade CTS é válida para que não haja reducionismos ao se tratar de questões científicas, tecnológicas e sociais. Completam que a utilização da sigla CTSA pretende conferir maior ênfase às questões ambientais para evitar um tratamento insuficiente dessa perspectiva na Educação em Ciências. De acordo com Santos (2011) parece apropriado o que esses autores propõem sobre a adoção de CTSA para que o ambiente tenha o foco adequado nesse novo modelo, porém, não cabe julgar o movimento CTS como ultrapassado, mas sim necessitando ser recontextualizado. É nesse sentido que questionamos a forma como essa inserção é feita no próprio termo CTS, com o Meio Ambiente (representado pela letra "A") possuindo uma dimensão comparável a Ciência, a Tecnologia e a Sociedade, quando o compreendemos como mais amplo e capaz de englobar todas essas dimensões. De qualquer forma, "[...] seja qual for o slogan a ser empregado é fundamental desvelar o significado do que queremos" (Id. Ibid., p. 38).

Em nossa análise verificamos que os autores utilizam de forma preponderante o termo CTS. Em números, 28 utilizaram CTS e apenas sete se remeteram a CTSA. Ressaltamos que os autores utilizam diferentes termos para caracterizar CTS/CTSA, como enfoque, abordagem, educação, relações, ênfase curricular, contexto, proposta e ensino. Analisar a utilização dos termos pode ser interessante dependendo do estudo/pesquisa que se objetiva. Em nosso caso, por exemplo, utilizamos o termo "Educação CTS" por entendermos que esse termo engloba a amplitude dos nossos objetivos com relação às produções CTS nordestinas, neste caso. Em outros contextos, a utilização é paritária por considerarmos um termo abrangente para caracterizar os propósitos de CTS no contexto educacional, pautado na problematização do currículo, do ensino, da formação de professores e da formação cidadã. Às vezes utilizar termos como enfoque ou abordagem, por exemplo, pode contribuir para a compreensão dessa perspectiva como uma metodologia ou até evidenciar aspectos que não são objetivos da Educação CTS. Mesmo 
assim, diversos pesquisadores atribuem significado aos termos de acordo com suas concepções e a direção que tomam as suas pesquisas.

Quanto aos trabalhos que se filiam à Educação CTSA, entendemos que, se a ideia é evitar reducionismos, é fundamental que haja uma justificativa e/ou explicação para o uso dessa nomenclatura. É certo que o número de pesquisas que se autodenominam CTSA ainda é reduzido (ABREU; FERNANDES; MARTINS, 2013), mas, destacamos que uma pesquisa foi desenvolvida na Bahia (no PPGEHFC), quatro no Rio Grande do Norte (no PPGECNM) e duas em Sergipe (no Programa de Pós-Graduação em Ensino de Ciências e Matemática - PPGECIMA). Notamos inclusive que Nunes (2014) em sua tese passa a utilizarse do termo CTS, porém, em sua dissertação (2010), aderiu ao termo CTSA.

As pesquisas que utilizam o termo CTS constituem $80 \%$ da produção na área quando comparadas às pesquisas que utilizam o termo CTSA. De fato, é muito comum utilizar esse termo e seus propósitos devido ao contexto sócio histórico de inserção no Brasil. É trivial visualizar trabalhos que utilizam o termo CTSA fazendo referência a publicações que, na verdade, utilizam o termo CTS, ou seja, utilizam como se fossem sinônimos. Entender e discutir, mesmo que de forma sucinta, os sentidos atribuídos pelos pesquisadores aos termos CTS/CTSA é importante para se evitar confusões teórico-metodológicas que contribuem para uma perigosa diversidade conceitual do campo, sendo muitas já descritas no contexto brasileiro.

\section{Contexto que se inserem as pesquisas}

O contexto em que se inserem as pesquisas sobre CTS mostram as tendências dessa perspectiva quanto a sua "implementação" nos diferentes níveis de ensino. Analisamos a partir dos resumos, e por vezes na metodologia, os contextos que as pesquisas se encontravam. Trazer um panorama quantitativo dessas características nos leva a compreender quais as necessidades regionais na visão dos pesquisadores nordestinos. Na tabela 1, apresentamos a distribuição das áreas específicas do contexto das pesquisas. 
TABELA 1. Contexto de desenvolvimento e/ou discussão das pesquisas

\begin{tabular}{lcc}
\hline \multicolumn{1}{c}{ CONTEXTO } & QUANTIDADE & $\%$ \\
\hline Ensino Fundamental & 2 & $6 \%$ \\
\hline Ensino Fundamental e Médio & 1 & $3 \%$ \\
\hline Ensino Médio & 11 & $31 \%$ \\
\hline Formação continuada de professores & 7 & $20 \%$ \\
\hline Formação continuada e Ensino Médio & 2 & $6 \%$ \\
\hline $\begin{array}{l}\text { Formação inicial de professores, de tecnólogos e de } \\
\text { bacharéis }\end{array}$ & 12 & $34 \%$ \\
\hline
\end{tabular}

Fonte: dados da pesquisa, 2017.

Podemos perceber que as pesquisas estão distribuídas em duas linhas amplas: ensino e aprendizagem e formação docente. Duas pesquisas incluídas nessa última linha discutem formação de professores e incluem também em suas pesquisas outros sujeitos, como estudantes de cursos de bacharelado e tecnólogos. A Educação CTS também se encontra relacionada a discussões sobre o currículo de Ciências e prevê a (re)organização deste. Entendemos que em qualquer possibilidade de implementação dessa proposta, o currículo será o alvo e as mudanças geradas estarão sempre ligadas a ele, seja para formar para a cidadania, para o desenvolvimento de valores e atitudes, para a tomada de decisão etc.

A partir do quadro notamos que os maiores índices das produções correspondem à formação inicial e continuada de professores $(54 \%)$ e ao Ensino Médio (31\%). Os processos formativos com enfoque na Educação CTS também são alvo de investigações em todo o Brasil (PRUDÊNCIO, 2013; SILVA, 2014; MÜNCHEN, 2016; ROSA; ARAÚJO, 2017). Essas pesquisas indicam a necessidade de trabalhar propostas para a formação de professores que agreguem referenciais críticos, no âmbito da Educação CTS, uma vez que existe uma carência de discussões sobre os pressupostos dessa perspectiva na formação docente.

Muitas pesquisas ainda relacionadas à formação de professores concentram-se em discussões sobre as concepções de Ciência, Tecnologia e Sociedade, com resultados que apontam para concepções que se enquadram em uma visão reducionista dessas três dimensões. Compreendemos que analisar as concepções dos licenciandos ou dos professores em exercício é o ponto inicial para se discutir sobre Educação CTS na formação de professores, mas defendemos que as pesquisas necessitam 
avançar para outros patamares, no sentido de promoverem processos formativos ou momentos de diálogo para um maior engajamento dos professores com essa perspectiva.

Ainda são comuns questionamentos do tipo "como ensinar de forma contextualizada?", "como ensinar para formar para cidadania", ou até mesmo "como ensinar com essa Educação CTS?" Essas indagações reclamam por uma maior instrumentalização docente a respeito das maneiras de se trabalhar com a Educação CTS em sala de aula, além de reivindicar a aplicabilidade das pesquisas da área no contexto educacional, especialmente nas escolas. Nesse sentido, nota-se que na região Nordeste esse propósito interventivo vem se expandido e isso ocorre não só pelo fato desse quantitativo de pesquisas já finalizadas, mas também por aquelas que estão em andamento nos próprios programas da região.

A quantidade de pesquisas na Educação Básica no contexto nordestino nos parece expressiva para os estados analisados, mas ainda se verifica uma concentração no Ensino Médio. Um dos motivos para isso, muito claro nas pesquisas brasileiras, deriva da formação dos pesquisadores, oriundos normalmente de cursos de licenciatura específicos (Biologia, Física, Química) e, portanto, com maior proximidade com as disciplinas do Ensino Médio. Talvez por conta disso também, seja verificada certa "facilidade" para inserir essa perspectiva nesse nível de ensino quando levamos em consideração a relação tema/conteúdo, ou para alguns, a relação conteúdo/tema. Assim, fica evidente que a maioria dos pesquisadores prefere trabalhar com os sujeitos nas disciplinas específicas do Ensino Médio ou, pelo menos com o último ano do Ensino Fundamental, quando os conhecimentos químicos e físicos são bem traçados. Desse modo, comumente nas pesquisas não só no Nordeste, mas no Brasil de maneira geral, encontramos relatos de pesquisas de natureza interventiva nas disciplinas de Biologia, Física, Química, Matemática etc., mesmo utilizando a denominação Ensino de Ciências.

Outros níveis de ensino ainda são pouco discutidos. Ferst e Ghedin (2014) ao realizarem um levantamento sobre a Educação CTS no contexto dos anos iniciais, constataram que houveram avanços de estudos dessa proposta 
direcionados para esse nível, no entanto, argumentam que a quantidade de pesquisas que tratam sobre essa temática nesse contexto tem um longo caminho a percorrer para sua possível efetivação no ensino.

Em relação às modalidades de ensino podemos citar a Educação de Jovens e Adultos (EJA) que, de forma crescente, passa a apresentar pesquisas relacionadas à Educação CTS (MIRANDA; TEIXEIRA, 2016). Isso é significativo para o Ensino de Ciências, uma vez que essa modalidade de educação ainda é bastante marginalizada e até ausente no processo de formação inicial na maioria dos cursos de licenciatura em Ciências (Biologia, Física e Química).

\section{Área do conhecimento da pesquisa na Educação Científica}

Elencamos essa última categoria com o intuito de analisar em que área a produção CTS no Nordeste se concentra. Estudar a área de conhecimento que a perspectiva CTS está atrelada é importante para delimitarmos os espaços em que esta ganha repercussão. É evidente que, muitos desses resultados estão relacionados à área de sua origem no contexto brasileiro e sabemos que os estudos sobre CTS no Brasil iniciaram no ensino de Química, seguido pelo ensino de Biologia e, depois, pelo de Física. No entanto, os pesquisadores do Ensino de Química e Física seguiram com a linha de pesquisa ainda no final da década de 1990 de forma mais intensa e tornaram-se referenciais da área até os dias atuais.

Esse processo de chegada da perspectiva CTS no contexto brasileiro e sua consequente repercussão no ensino de Química gerou inúmeras pesquisas nessa área. Hoje a Educação Química é a área do conhecimento em Ciências que mais tem materiais na perspectiva CTS. Wildson Santos, em coautoria com outros pesquisadores, articulou projetos que originaram livros didáticos ${ }^{11}$ de Química com base em aspectos socientíficos que potencializam as interações dialógicas em sala de aula, facilitando a emergência de situações vivenciais dos alunos e o desenvolvimento de atitudes e valores em uma perspectiva humanística (SANTOS; MORTIMER, 2009). Com esse material, Wildson Santos e Gerson Mól, ainda na década de 1990, procuraram por meio da

11 Química na sociedade (1998), Química e Sociedade (2003) e Química cidadã (2010, 2013, 2016). 
contextualização temática, introduzir textos que problematizam a realidade dos sujeitos e contribuem para a formação da cidadania (SANTOS, 2007).

O ensino de Física e Biologia possuem poucos materiais didáticos, entretanto, assim como no ensino de Química, existem expressivas pesquisas e pesquisadores engajados com o desenvolvimento dessa perspectiva. A Educação Matemática não possui muitos trabalhos nessa temática. No entanto, essa área começa a se desvincular do Ensino de Ciências com a criação de novos programas e eventos específicos. Segundo Araújo-Queiroz (2016), os autores de livros didáticos de Biologia vêm se esforçando para inserirem textos, infográficos e atividades que contemplem aspectos da Educação CTS, mesmo assim, acabam por adotar uma listagem de conteúdos tradicionais irredutíveis e na maioria das vezes desconectados da prática social dos sujeitos.

Dessa forma, na região Nordeste a perspectiva CTS encontra-se heterogênea entre as áreas de conhecimento. Contabilizamos 13 pesquisas no ensino de Química, 10 no ensino de Biologia, 6 no ensino de Física, 2 na Educação Matemática e 4 em Ciências Naturais sem especificar uma área. $\mathrm{Na}$ figura 2, apresentamos esse quantitativo em porcentagem que se relaciona também à produção por estado e ao PPG.

FIGURA 2. Representação da produção CTS por área do conhecimento

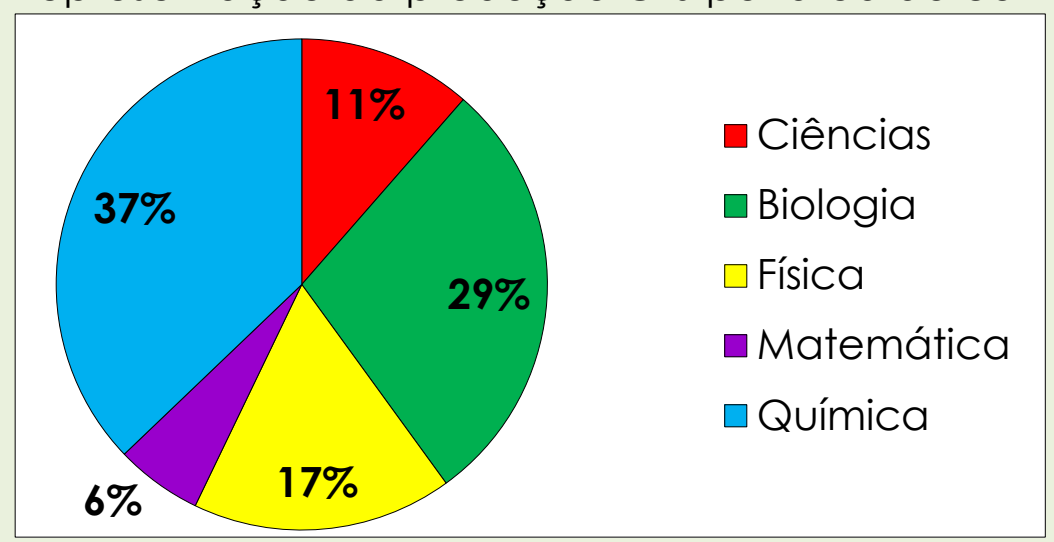

Fonte: dados da pesquisa, 2017.

Na Bahia a maioria das pesquisas concentram-se no ensino de Biologia, enquanto que em Pernambuco e Sergipe prevalece a Química, na Paraíba a discussão sobre CTS ocorre no âmbito do ensino de Física e Matemática e no Rio Grande do Norte essas pesquisas perpassam o ensino de Química, Física e 
Biologia. Isso nos mostra que cada estado possui um núcleo de professores que investigam sobre CTS em determinada área de concentração e que se constituem em uma rede de pesquisadores oriundos de grupos de pesquisas consolidados no Brasil, seja da área de ensino ou não.

Por exemplo, a professora Edenia Amaral (Ensino de Química - UFRPE) foi orientada no doutorado por Eduardo Mortimer no mesmo período que Mortimer orientava Wildson Santos, juntos, realizaram pesquisas no Brasil e no exterior com a Educação CTS, no momento em que essa perspectiva ganhava grande repercussão. Posteriormente ela orientou a primeira dissertação de CTS no Nordeste no período de 2005-2007. Isso revela a importância da relação dos grupos de pesquisa brasileiros na dispersão dos seus pesquisadores, mais uma vez, reforçando a migração de professores de outras regiões para o Nordeste.

No ensino de Biologia a perspectiva CTS é expressivamente discutida na Bahia. O grupo de pesquisa do professor Paulo Marcelo Marini Teixeira, da UESB, discute a Educação CTS a partir de uma visão progressista, tendo como um de seus referenciais a pedagogia histórico-crítica de Demerval Saviani, algo ainda não muito usual na Educação CTS, de forma geral. Já nos quatro trabalhos de dissertação em desenvolvimento na Universidade Estadual de Santa Cruz UESC, em llhéus, essa perspectiva também está ancorada em um viés progressista, porém, incorporando os ideais educacionais de Paulo Freire.

Como vimos, ainda são necessários avanços para o ensino de Física e a Educação Matemática, bem como o Ensino de Ciências no nível fundamental no que tange ao trabalho com a Educação CTS. Essas lacunas devem se constituir como pontos estratégicos para esse campo de estudo, sinalizando para os pesquisadores da área possibilidades reais de desenvolvimento de novas pesquisas empíricas nesses contextos formativos.

\section{ALGUMAS CONSIDERAÇÕES}

As inquietações para a publicação de um trabalho como este sempre estiveram dilatadas em nossas leituras. Isso porque entendemos que, apesar de ainda haver muito a ser definido na Educação CTS, é preciso avanços na discussão de modo que as inter-relações CTS sejam introduzidas nas pesquisas não só como um campo de estudo, mas, como um campo necessário e 
emergente para formação humana a partir do atendimento das nossas necessidades regionais.

Esse levantamento nos traz também preocupações em relação aos estados da região Nordeste que não discutem sobre a perspectiva CTS. Ao mesmo tempo, nos incomoda o fato de que nos estados onde são encontrados trabalhos com essa temática, a maioria desses, mesmo estando finalizados, ainda não foram publicados em periódicos da área de Educação em Ciências. A nosso ver essa situação tende a colaborar para que as pesquisas nordestinas permaneçam em um quadro de exclusão no panorama da produção sobre Ensino de Ciências no contexto brasileiro, principalmente nesse campo que nos propomos a analisar. É necessário um estudo apurado para o entendimento desses direcionamentos dos resultados de pesquisas. Além disso, seria necessário mapear os pesquisadores que estão engajados com estudos CTS para estabelecermos relações de pesquisa a partir desses. É preciso, portanto, publicar, divulgar e contribuir com os resultados das pesquisas realizadas em âmbito regional.

Mais importante que os dados quantitativos desse levantamento são as discussões e os direcionamentos que as novas pesquisas a partir daqui podem tomar. Tornar os PPG reconhecidos por suas linhas teóricas de pesquisa no Nordeste é delegado aos pesquisadores credenciados e vinculados a estes, bem como aos pós-graduandos. Perguntas sobre onde está CTS no Nordeste, porque a maior concentração de pesquisas ainda é nas regiões no Sul e Sudeste, ou ainda sobre o motivo da falta de referenciais locais que não devem permanecer somente em nossos discursos, pois como vimos essa região possui uma produção em Educação CTS passível de ser utilizada nas pesquisas em ensino. Dessa forma, já é hora de começarmos a utilizar como referenciais o desenvolvimento de propostas CTS dos pesquisadores dessa região.

O campo caminha para um processo de consolidação regional, mas, infelizmente, os estados Maranhão, Piauí, Ceará e Alagoas ainda apresentam uma carência de pesquisas nessa temática. Constatamos então que é preciso conhecer a rede de pesquisadores que trabalham com CTS no Brasil, mesmo em áreas de concentração distintas e analisar o desenvolvimento das 
pesquisas na formação de graduandos e pós-graduandos. Além disso, considerando as limitações dessa pesquisa, seria interessante analisar o porquê da maioria dos trabalhos acadêmicos realizados em PPG da região Nordeste não estarem publicados em periódicos da área.

Uma possível limitação regional está no fato das universidades nordestinas ainda não comportarem eventos científicos específicos da área, como o Encontro Nacional de Pesquisa em Educação em Ciências - ENPEC e os Encontros Nacionais de Ensino de Biologia, Física e Química, o que tem dificultado a participação dos pesquisadores dessa região. Porém, não podemos negar que a pós-graduação avança em relação aos estudos com essa temática na região que, sem dúvidas, tende a estar muito presente nos próximos anos em periódicos conhecidos na área de ensino no Brasil.

Muitos ainda criticam as pesquisas que apresentam suas inquietações e as discutem a partir de levantamentos/pesquisas bibliográficas. Esse tipo de concepção errônea reforça uma visão deformada sobre essa forma de obtenção e análise de informações. Ao contrário, acreditamos na relevância desse tipo de pesquisa e sua importância para caracterizar o estado de discussões e difusões sobre a pesquisa em Educação CTS, sob o cuidado de não contribuir para relevar a polissemia presente no campo.

Ainda há muito por se fazer, principalmente nesse momento que a perspectiva CTS está em processo de expansão e ainda hoje buscando delimitar possibilidades metodológicas para a implementação de práticas de ensino no contexto educacional. Analisar a distribuição geográfica das pesquisas sobre essa temática, possibilita a identificação de onde estão situados os principais pesquisadores, grupos de pesquisa e PPG da área que contribuem para o avanço desse campo de conhecimento, além de trazer dados e olhares atenciosos para a realidade a qual estamos inseridos e, possivelmente, contribuir para uma rede de contatos entre esses pesquisadores. Deste modo, defendemos a formação da cidadania, o desenvolvimento de valores e atitudes e a tomada de decisão por meio de práticas com essa perspectiva, fazendo-se válida como um amplo campo de pesquisa para a pós-graduação no Brasil. 


\section{REFERÊNCIAS}

ABREU, T. B.; FERNANDES, J. P.; MARTINS, I. Levantamento sobre a produção CTS no Brasil no período de 1980-2008 no campo de ensino de ciências. Alexandria (UFSC), v. 6, n. 2, p. 3-32, 2013.

AIKENHEAD, G. STS Education: A Rose by Any Other Name. In: Cross, R. (Ed.): A Vision for Science Education: Responding to the work of Peter J. Fensham, p. 5975. New York: Routledge Falmer, 2003.

ALMEIDA, M. K. S. X. Física térmica com ênfases curriculares em CTSA e ensino por investigação. Dissertação - Universidade Federal do Rio Grande do Norte, Natal, 2016.

ANDRADE JÚNIOR, J. A. Episódios históricos no contexto do ensino de ciências: a energia nuclear e sua utilização. Dissertação - Universidade Estadual da Paraíba, Campina Grande, 2015.

ANDRADE, M. A. S. Construção e aplicação de uma sequência didática colaborativa a partir de uma questão sociocientífica sobre agrotóxicos na perspectiva CTSA. Dissertação - Universidade Federal da Bahia, Salvador, 2016. ARAÚJO-QUEIROZ, M. B. Análise da perspectiva curricular CTS em livros didáticos de Biologia: enfoques, finalidades e confluências. Trabalho de Conclusão de Curso (Monografia) - Universidade Federal do Piauí, Piauí, 2016.

ARAÚJO-QUEIROZ, M. B.; MENDES, F. C.; ARAUJO, W. S. Estudos CTS no Ensino de Biologia: uma revisão com base em anais de eventos científicos. Revista da SBEnBIO, v. 9, p. 4974-4983, 2016.

AULER, D. Articulação entre pressupostos do educador Paulo Freire e do movimento CTS: novos caminhos para a educação em ciências. Contexto \& Educação, v. 1, n77, p. 167-188, 2007.

AULER, D. Interações entre Ciência-Tecnologia-Sociedade no Contexto da Formação de Professores de Ciências. Tese (Doutorado em Educação) Universidade Federal de Santa Catarina, Florianópolis: CED/UFSC, 2002.

AULER, D.; DELIZOICOV, D. Alfabetização científico-tecnológica para quê? Ensaio: Pesquisa em Educação em Ciências, v. 3, n. 2, p. 122-134, 2001.

BAROLLI, E.; VILLANI, A. A formação de professores de Ciências no Brasil como campo de disputas. Revista Exitus, v. 5, n. 1, p. 72-90, 2015.

BEZERRA, M. E. B. Um estudo sobre o ensino de evaporação no contexto

Ciência, Tecnologia e Sociedade. Dissertação - Universidade Federal do Rio Grande do Norte, Natal, 2014.

BINATTO, P. F. Enfoque Ciência, Tecnologia e Sociedade na Formação Reflexiva de Futuros Professores de Biologia: Possibilidades, Desafios e Contribuições. Dissertação - Universidade Estadual do Sudoeste da Bahia, Jéquié, 2015.

BITENCOURT, I. M. A Botânica no ensino médio: análise de uma proposta didática baseada na abordagem CTS. Dissertação - Universidade Estadual do Sudoeste da Bahia, Jéquié, 2013. 
COSTA, A. L. A. Matemática financeira e cidadania: interlocução, leituras e experiências. Dissertação - Universidade Estadual da Paraíba, Campina Grande, 2015.

DAGNINO, R.; THOMAS, H.; DAVYT, A. El Pensamiento em Ciencia, Tecnología y Sociedad em Latinoamérica: uma interpretación política de su trayectoria. In: DAGNINO, R.; THOMAS, H. Ciência, Tecnologia e Sociedade: uma reflexão latino-americana. Taubaté: Cabral Editora e Livraria Universitária, 2003.

DANTAS, J. M. O cinema e o ensino da física: uma experiência sob olhar CTS. Dissertação - Universidade Estadual da Paraíba, 2011.

FAILACHE, M. V.; MOTTA, P. C.; SOBRAL, V. C. S.; BATISTA, Z. N; MIOTELLO, V. A perspectiva de estudos em ciência, tecnologia e sociedade (CTS) no Nordeste brasileiro - um estudo preliminar sobre o desenvolvimento do enfoque ciência, tecnologia e sociedade na pós-graduação. Revista Científica Interdisciplinar Interlogos, Paranaguá, v1, n. 1. jan, p. 19-47, 2017.

FERST, E. M.; GHEDIN, E. Panorama das publicações nos ENPECs sobre CTS nos anos iniciais do ensino fundamental. Anais... In: IV Simpósio Nacional de Ensino de Ciências e Tecnologia, Ponta Grossa-PR, 2014.

FIRME, R. N. A abordagem ciência-tecnologia-sociedade (CTS) no ensino da termoquímica: análise da construção discursiva de uma professora sobre conceitos científicos. Tese (Doutorado em Educação) - Universidade Federal de Pernambuco, Recife, 2012.

FIRME, R. N. A implementação de uma abordagem CTS (ciência-tecnologiasociedade) no ensino da química: um olhar sobre a prática pedagógica. Dissertação - Universidade Federal Rural de Pernambuco, 2007.

FREITAS, L. M.; GHEDIN, E. Pesquisas sobre estado da arte em CTS: análise comparativa com a produção em periódicos nacionais. Alexandria (UFSC), v. 8, n. 3, p. 3-25, 2015.

GOMES, R. R. A. Musicalizando a Biologia: exploração de letras musicais sob a perspectiva CTSA na formação inicial docente. Dissertação - Universidade Federal do Rio Grande do Norte, Natal, 2016.

GONÇALVES, P. G. F; SILVA, G. N. As pesquisas sobre Ciência, Tecnologia e Sociedade no Nordeste: um retrato das dissertações na área de ensino de ciências naturais e matemática. Conexões: Ciência e Tecnologia, v. 10, n. 4, p. $42-50,2016$.

JESUS, M. P. Contextualização do ensino de química por meio do enfoque CTS atrelado a pedagogia de Paulo Freire. Dissertação - Universidade Federal de Sergipe, Aracajú, 2017.

LIMA NETO, J. A. O uso da abordagem CTSA no ensino de energia tendo o desenvolvimento sustentável como eixo temático. Dissertação - Universidade Federal do Rio Grande do Norte, Natal, 2012.

MACEDO, F. L. Biogás: discutindo as relações CTS com os alunos do ensino médio. Dissertação - Universidade Federal do Rio Grande do Norte, Natal, 2014. 
MARQUES, A. M. Açude do cais: uma proposta de aplicação de uma sequência de atividades didáticas em um contexto real. Dissertação Universidade Federal do Rio Grande do Norte, Natal, 2014.

MIRANDA, M. L. O.; TEIXEIRA, P. M. M. A articulação da tríade CTS: reflexões sobre o desenvolvimento de uma proposta didática aplicada no contexto da EJA. Investigações em Ensino de Ciências (Online), v. 21, p. 124-144, 2016.

MÜNCHEN, S. A inserção da perspectiva Ciência-Tecnologia-Sociedade na formação inicial de professores de Química. Tese (Doutorado em Educação) Universidade Federal de Santa Maria, Rio Grande do Sul, 2016.

NUNES, A. O. Abordando as relações CTSA no ensino da química a partir das crenças e atitudes de licenciandos: uma experiência formativa no Sertão Nordestino. Dissertação - Universidade Federal do Rio Grande do Norte, Natal, 2010.

NUNES, A. O. Possibilidades de enfoque CTS para o ensino superior de química: proposta de uma abordagem para ácidos e bases. Tese (Doutorado em Química) - Universidade Federal do Rio Grande do Norte, Natal, 2014.

OLIVEIRA, A. L. P. Uma sequencia didática a partir da temática terremotos com ênfase em CTS. Dissertação - Universidade Federal do Rio Grande do Norte, Natal, 2012.

OLIVEIRA, F. R. F. Vídeo e ensino de ciências: um olhar CTS sobre a produção dos alunos. Dissertação - Universidade Estadual da Paraíba, Campina Grande, 2010.

OLIVEIRA, T. B. Ensino de ciências na perspectiva CTS: concepções e práticas escolares. Dissertação - Universidade Federal de Sergipe, 2013.

PINTO, S. L.; GOULART, A. O. F.; VERMELHO, S. C. S. D. O tema CTS nas pesquisas sobre ensino de ciências: revisão bibliográfica dos últimos 25 anos. Revista da SBEnBio, n. 9, p. 5311-5321, 2016.

PORTO, M. L. O. O ensino de Biologia na Educação de Jovens e Adultos (EJA) por meio do enfoque Ciência - Tecnologia - Sociedade (CTS): Análise de uma proposta desenvolvida. Dissertação - Universidade Estadual do Sudoeste da Bahia, Jéquié, 2014.

PRUDENCIO, C. A. V. Perspectiva CTS em estágios curriculares em espaços de divulgação científica: contributos para a formação inicial de professores de Ciências e Biologia. Tese (Doutorado em Educação) - Universidade Federal de São Carlos, São Carlos, 2013.

ROSA, I. S. C. Abordagem CTSA no ensino de ecologia: uma contribuição para a formação de cidadãos críticos. Dissertação - Universidade Federal de Sergipe, Aracajú, 2014.

ROSA, S. E.; ARAÚJO, W. S. Processos Formativos no Contexto Brasileiro: uma articulação dos pressupostos de Freire e a Educação CTS. XI Encontro Nacional de Pesquisa em Educação em Ciências. Anais... Atas do XI ENPEC, 2017. 
SANTANA, D. C. O. O uso de texto de divulgação científica em uma unidade de ensino com uma abordagem CTS para educação química. Dissertação Universidade Federal do Rio Grande do Norte, Natal, 2016.

SANTANA, T. A. Aplicação do enfoque CTS no ensino de Bioquímica: análise de uma experiência didática. Dissertação - Universidade Estadual do Sudoeste da Bahia, Jéquié, 2014.

SANTIAGO, J. F. A. O uso de textos de divulgação científica como recurso didático em aulas de biologia: concepções e relações com a abordagem CTS de ensino. Dissertação - Universidade Federal do Rio Grande do Norte, Natal, 2016.

SANTOS, A. O. Atividade experimental de condutividade eletrolítica: uma abordagem da química com base em uma proposta CTSA. Dissertação Universidade Federal de Sergipe, Aracajú, 2015.

SANTOS, E. P. Concepções dos licenciandos em Química da Universidade Federal de Sergipe (UFS) sobre a contextualização crítica numa perspectiva de ensino CTS. Dissertação - Universidade Federal de Sergipe, Aracajú, 2015.

SANTOS, M. E. M. Concepções sobre Ciência, Tecnologia e Sociedade e concepções de ensinar de futuros professores de ciências inseridos em um projeto baseado em Arranjos Produtivos Locais. Dissertação - Universidade Federal de Sergipe, Aracajú, 2017.

SANTOS, R. C. S. Ciência-Tecnologia-Sociedade: suas interrelações e seu ensino nas concepções de licenciandos em Química de uma universidade da rede privada da cidade de Aracaju. Dissertação - Universidade Federal de Sergipe, Aracajú, 2017.

SANTOS, W. L. P. Contextualização no ensino de ciências por meio de temas CTS em uma perspectiva crítica. Ciência \& Ensino, v. 1, p. 1-12, 2007.

SANTOS, W. L. P. Educação científica humanística em uma perspectiva Freireana: resgatando a função do ensino de CTS. Alexandria (UFSC), v. 1, p. 109-131, 2008.

SANTOS, W. L. P. Educação CTS e Cidadania: Confluências e Diferenças. Amazônia (UFPA), v. 9, p. 49-62, 2012.

SANTOS, W. L. P. Scientific literacy: a Freirean perspective as a radical view of humanistic science education. Science Education (Salem, Mass. Print), v. 93, p. 361-382, 2009.

SANTOS, W. L. P. Significados da educação científica com enfoque CTS. In SANTOS, Wildson, L. P.; Auler, Décio (Orgs.). CTS e Educação Científica: desafios, tendências e resultados de pesquisas. Brasília: Editora Universidade de Brasília, 2011, p. 21-47.

SANTOS, W. L. P.; MORTIMER, E. F. Abordagem de aspectos sociocientíficos em aulas de ciências: possibilidades e limitações. Investigações em Ensino de Ciências, v. 14, n. 2, p. 191-218, 2009. 
SANTOS, W. L. P.; MORTIMER, E. F. Tomada de decisão para ação social responsável no ensino de ciências. Ciência \& Educação (Bauru), v. 7, n. 1, p. 95$111,2001$.

SANTOS, W. L. P.; MORTIMER, E. F. Uma análise de pressupostos teóricos da abordagem C-T-S (Ciência - Tecnologia - Sociedade) no contexto da educação brasileira. Ensaio: Pesquisa em Educação em Ciências, v. 02, n. 02, p. 01-23, 2000.

SILVA, B. H. A perspectiva CTS na formação inicial de professores de química: construindo subsídios para uma ação didático-pedagógica inovadora. Dissertação - Universidade Federal Rural de Pernambuco, 2014.

SILVA, D. P. As dimensões ética e científica na formação para tomada de decisão sobre uso de animais nas Ciências em um contexto de Educação CTS. Dissertação - Universidade Federal da Bahia, Salvador, 2016.

SILVA, E. M. L.; MOREIRA, M. R.; SILVA, C. A. C. F.; SILVA, E. V. Produções em abordagem CTS/CTSA no ensino de ciências com ênfase na formação docente. Anais... III Congresso Internacional das Licenciaturas, 2016.

SILVA, M. A. A. Ciência, tecnologia e sociedade, experimentação e formação inicial de professores de química: explorando possibilidades. Dissertação Universidade Estadual do Sudoeste da Bahia, Jéquié, 2016.

SILVA. D. J. R. Abordagem CTS e ensino de matemática crítica: um olhar sobre a formação inicial dos futuros docentes. Dissertação - Universidade Estadual da Paraíba, Campina Grande, 2012.

SOUSA, G.P. Educação CTS e Genética - elementos para a sala de aula: potencialidades e desafios. Dissertação - Universidade Estadual do Sudoeste da Bahia, Jéquié, 2013.

SOUZA, A. M. Despertando responsabilidade social no ensino médio por meio de temáticas associadas à física nuclear. Dissertação - Universidade Federal do Rio Grande do Norte, Natal, 2010.

STRIEDER, R. B. Abordagem CTS na Educação Científica no Brasil: Sentidos e Perspectivas. Tese (Doutorado em Ensino de Ciências) - Instituto de Física e Faculdade de Educação, Universidade de São Paulo, São Paulo, 2012.

STRIEDER, R. B; KAWAMURA, M. R. D. Educação CTS: parâmetros e propósitos brasileiros. Alexandria: Revista de Educação em Ciência e Tecnologia, v. 10, n.

1, p. 27-56, 2017.

VILCHES, A; GIL PÉREZ, D. PRAIA, J. De CTS a CTSA: educação por um futuro sustentável. In SANTOS, Wildson, L. P.; Auler, Décio (Orgs.). CTS e Educação Científica: desafios, tendências e resultados de pesquisas. Brasília: Editora Universidade de Brasília, 2011, p. 161-184.

Recebido em: Novembro de 2017 Aprovado em: Maio de 2018 